\title{
Accuracy of the Fetal Echocardiogram in Double-outlet Right Ventricle
}

\author{
Sarah Gelehrter, MD, Sonal T. Owens, MD, Mark W. Russell, MD, Mary E. van der Velde, MD, and \\ Carlen Gomez-Fifer, MD
}

Michigan Congenital Heart Center, University of Michigan, Ann Arbor, Mich, USA

\begin{abstract}
A B S T R A C T
Objective. Double-outlet right ventricle (DORV) is a complex congenital heart defect with heterogeneous anatomy. Patients require a variety of surgical interventions, and the long-term prognosis is variable. Therefore, accurate prenatal diagnosis is important in providing parents with appropriate counseling.

Design. Medical records were reviewed in patients with a diagnosis of DORV who had fetal echocardiography at our institution from 1998 to 2004. Pre- and postnatal diagnoses were compared, anticipated surgical procedure was compared with surgery performed, and neonatal outcome was assessed.

Results. The study group consisted of 49 fetal patients with 6 in utero deaths (including 4 terminations), 2 patients lost to follow-up, and 41 live births. Postnatal echocardiograms or autopsy results were available on 42 patients. The overall accuracy of fetal echocardiography in making a correct diagnosis of DORV was $76 \%$. Accurate prenatal prediction of the type of cardiac surgery that would be performed was made in $91 \%$ of patients who had surgery. Among live births, survival to hospital discharge was $76 \%$. Survival was $50 \%$ in patients with extracardiac or chromosomal anomalies, compared with $92 \%$ in infants without additional anomalies.

Conclusion. In most cases, careful evaluation by fetal echocardiography can determine essential anatomic details in fetuses with DORV with enough accuracy to allow for accurate counseling regarding the type of surgery needed. Survival is better than previously reported but is poor in patients with extracardiac or chromosomal abnormalities.
\end{abstract}

Key Words. Fetal Echocardiography; Double-outlet Right Ventricle; Congenital Heart Disease

\section{Introduction}

$\mathrm{T}$ he diagnosis of double-outlet right ventricle (DORV) encompasses a broad spectrum of complex congenital heart defects with heterogeneous anatomy characterized by both great arteries arising predominantly from the morphological right ventricle. This spectrum includes variants similar to an isolated subaortic ventricular septal defect (VSD), those similar to tetralogy of Fallot, and variants similar to $\mathrm{D}$-transposition of the great arteries. DORV can also be a part of complex single-ventricular anatomy, including heterotaxy, and can be seen in association with ventricular inversion. Because of this variability, patients may require a variety of surgical repairs or palliations with very different short- and long-term prognoses.

Prenatal detection of congenital heart disease has significantly increased in the past decade. ${ }^{1,2}$
This is likely due to improved obstetric awareness of the diversity of heart lesions, as well as increased emphasis on screening for outflow tract abnormalities and other subtle details. Advances in fetal echocardiographic technology and training have also contributed to enhanced detection of the anatomic details in complex cardiac defects such as DORV.

Despite this trend of improvement in fetal cardiac diagnosis, previous studies evaluating fetal diagnosis of DORV report low accuracy of diagnosis in this particular group. ${ }^{3-5}$ In addition, studies have shown that patients with conotruncal malformations were the most likely to have errors in fetal diagnosis. ${ }^{2}$ Of patients with conotruncal abnormalities, most of the diagnostic errors occurred in the group with DORV.

The importance of accuracy in obtaining diagnostic details is not purely academic. Accurate prenatal diagnosis is crucial in providing appropriate

(C) 2007, the Authors Congenit Heart Dis. 2007;2:32-37
Journal compilation @ 2007, Blackwell Publishing, Inc. 
counseling to parents of fetuses with DORV. Given the broad spectrum of possible variants, associated lesions, resultant surgical interventions, and predicted outcomes, counseling must be tailored to each individual case.

The purpose of this study is to determine whether improvements in fetal echocardiography can result in increased accuracy of diagnosis of DORV and, more importantly, more accurate prediction of the type of surgery needed. We hypothesize that this can be accomplished through careful determination of essential anatomic details such as VSD location and size, great artery orientation and size, and ventricular size.

\section{Methods}

The fetal and echocardiographic databases at our institution were searched for patients from 1998 to 2004 with a fetal diagnosis of DORV, or a postnatal diagnosis of DORV with an alternative fetal diagnosis. Medical records were reviewed to compare the fetal echocardiographic diagnosis to the postnatal diagnosis made by echocardiography, surgery, or autopsy. The echocardiographic and surgical diagnoses were in agreement in almost all cases; in the remaining cases, the intracardiac anatomy was not fully evaluated at the time of surgery and the echocardiographic diagnosis was used. We assessed the agreement between fetal echocardiography and postnatal evaluation in determining VSD location, great artery orientation and size, and ventricular size. The type of surgical repair predicted prenatally, as documented in the clinic letter by the cardiologist performing the fetal echocardiogram, was compared with the surgery performed. In addition, we noted the presence of any major extracardiac or chromosomal anomalies to determine their effect on neonatal outcome.

A 2-tailed Fisher exact test was used to examine for differences in frequency data between groups. We defined statistical significance as a $P$ value $<.05$.

\section{Results}

The study group consisted of 49 patients with complete fetal cardiac evaluation. The median gestational age at first fetal study at our institution was 29.5 weeks of gestation (range: 19-38 weeks). The mean number of fetal echocardiograms performed on each patient at our institution was 2.2 (range: 1-5). There were 6 in utero deaths (includ- ing 4 pregnancy terminations), and 2 patients lost to follow-up. Postnatal echocardiograms or autopsy results were available on 42 patients, including 4 patients with in utero demise and 38 live-born patients. Three live-born patients had no postnatal cardiac evaluation because of extensive extracardiac disease and early postnatal demise.

Prenatal and postnatal diagnoses are presented in Figure 1. Among the 40 fetuses with prenatal diagnosis of DORV, 23 had ventricular hypoplasia: 3 with hypoplastic right and 20 with hypoplastic left ventricle. Seventeen patients were diagnosed with normal biventricular size: 6 with normally related great arteries, 10 with D-malposed great arteries, and 1 with L-malposed great arteries. There were 2 additional patients who were found to have DORV as part of their diagnoses only on postnatal evaluation. Seventeen patients had major extracardiac or chromosomal anomalies; the most common variant of DORV seen in patients with anomalies was DORV with hypoplastic left ventricle, but at the same frequency as in the population as a whole.

The overall accuracy of fetal diagnosis was $76 \%$; 32 of the 42 patients with postnatal evaluation available were correctly diagnosed with DORV prenatally. Two patients with complex single-ventricle lesions were found to have DORV as part of the lesion only at postnatal evaluation. Heterotaxy syndrome was seen in $29 \%$ of patients and there were 10 patients with unbalanced atrioventricular canal defects. Eight patients had an incorrect fetal diagnosis of DORV. All were diagnosed postnatally with lesions that are anatomically similar to a subtype of DORV. An accurate diagnosis of DORV was made in $84 \%$ of cases with single-ventricular anatomy, and $65 \%$ of those with 2 ventricular anatomy. In all the cases of in utero death where autopsy results were available, an accurate diagnosis of DORV with hypoplastic left ventricle had been made prenatally. The sensitivity of a fetal diagnosis of DORV was $94 \%$ with a positive predictive value of $80 \%$. We found no differences in gestational age at first study or number of studies performed between those with accurate diagnosis and those with missed diagnoses.

The accuracy of the fetal echocardiogram in determining cardiac details is shown in Table 1. For the entire group, regardless of eventual postnatal diagnosis, accurate prenatal diagnosis of all anatomic details (ventricular adequacy, great artery orientation, great artery size, and VSD location) was made in $60 \%$ of cases. The sensitivity 


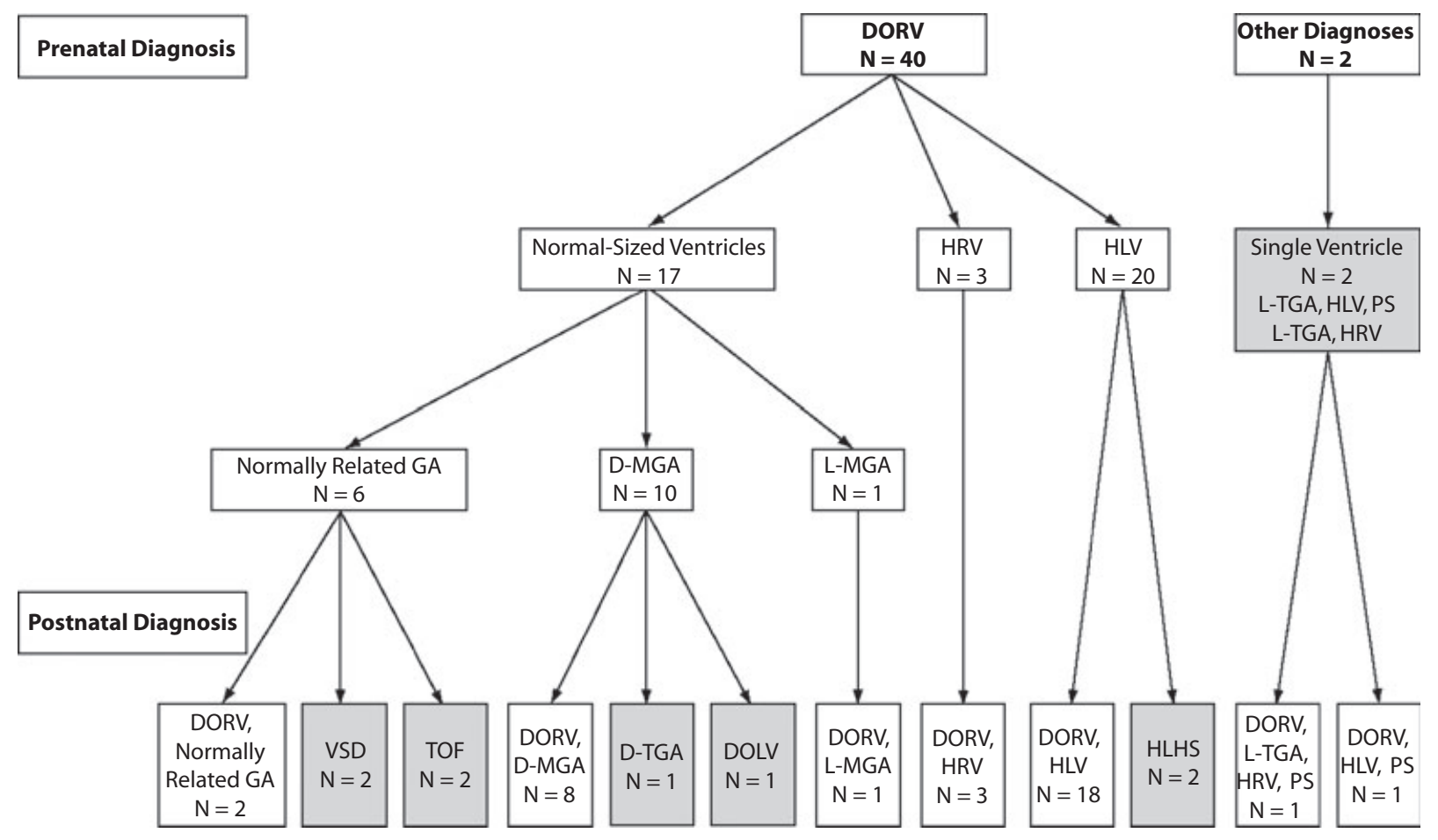

Figure 1. Pre- and postnatal diagnoses of infants with postnatal follow-up. Shaded boxes represent discrepancies between pre- and postnatal diagnoses. DOLV indicates double-outlet left ventricle; DORV, double-outlet right ventricle; D-MGA, Dmalposed great arteries; D-TGA, D-transposition of the great arteries; GA, great arteries; HLHS, hypoplastic left heart syndrome; HLV, hypoplastic left ventricle; HRV, hypoplastic right ventricle; L-MGA, L-malposed great arteries; L-TGA, Ltransposition of the great arteries; PS, pulmonary stenosis; TOF, tetralogy of Fallot; VSD, ventricular septal defect.

Table 1. Accuracy of Fetal Diagnosis

\begin{tabular}{lllllll}
\hline & \multicolumn{7}{l}{ Anatomic Details } \\
\cline { 2 - 6 } Fetal Diagnosis $(\mathbf{n})$ & Diagnosis of DORV & All Details & VSD Location & GA Size & GA Orientation & Ventricular Size \\
\hline All Patients (42) & $32(76)$ & $25(60)$ & $33(79)$ & $33(79)$ & $34(81)$ & $41(98)$ \\
Hypoplastic ventricle (25) & $21(84)$ & $10(40)$ & $17(68)$ & $16(64)$ & $17(68)$ & $25(100)$ \\
Normal-sized ventricles (17) & $11(65)$ & $15(88)$ & $16(94)$ & $17(100)$ & $17(100)$ & $16(94)$ \\
$P$ value & .28 & $.003^{*}$ & .06 & $.03^{*}$ & $.01^{*}$ & .40 \\
\hline
\end{tabular}

Number (\%) of patients with correct diagnosis or accurate determination of specific anatomic details.

* Statistically significant $P$ value comparing hypoplastic ventricle to normal sized ventricle patients using Fisher's exact test.

DORV indicates double-outlet right ventricle; GA, great artery; VSD, ventricular septal defect.

and specificity of prenatal detection of arch obstruction were both $88 \%$ with a negative predictive value of $97 \%$. Detailed anatomic evaluation was significantly more accurate in patients with 2 ventricular anatomy than in those with single-ventricle hearts $(P=.003)$.

We also examined the ability to predict for the need for ductal patency after birth to support either the pulmonary or systemic circulations. Twenty-two patients were predicted prenatally to possibly or definitely need prostaglandin infusion after delivery; $100 \%$ of those predicted to be definitely ductal dependent were predicted correctly and $62 \%$ of those predicted to possibly need pros- taglandin infusion were found to be ductal dependent. Only 1 patient predicted not to be ductal dependent was found postnatally to have a discrete coarctation that required prostaglandin infusion. The need for postnatal prostaglandin infusion could be predicted by fetal echocardiogram with a sensitivity of $96 \%$, a specificity of $62 \%$, a positive predictive value of $81 \%$, and a negative predictive value of $89 \%$.

Thirty-three patients in our study population had surgery (Figure 2). The surgical procedures performed were grouped into 4 categories: (1) VSD closure with or without relief of right ventricular outflow tract obstruction; (2) VSD closure 


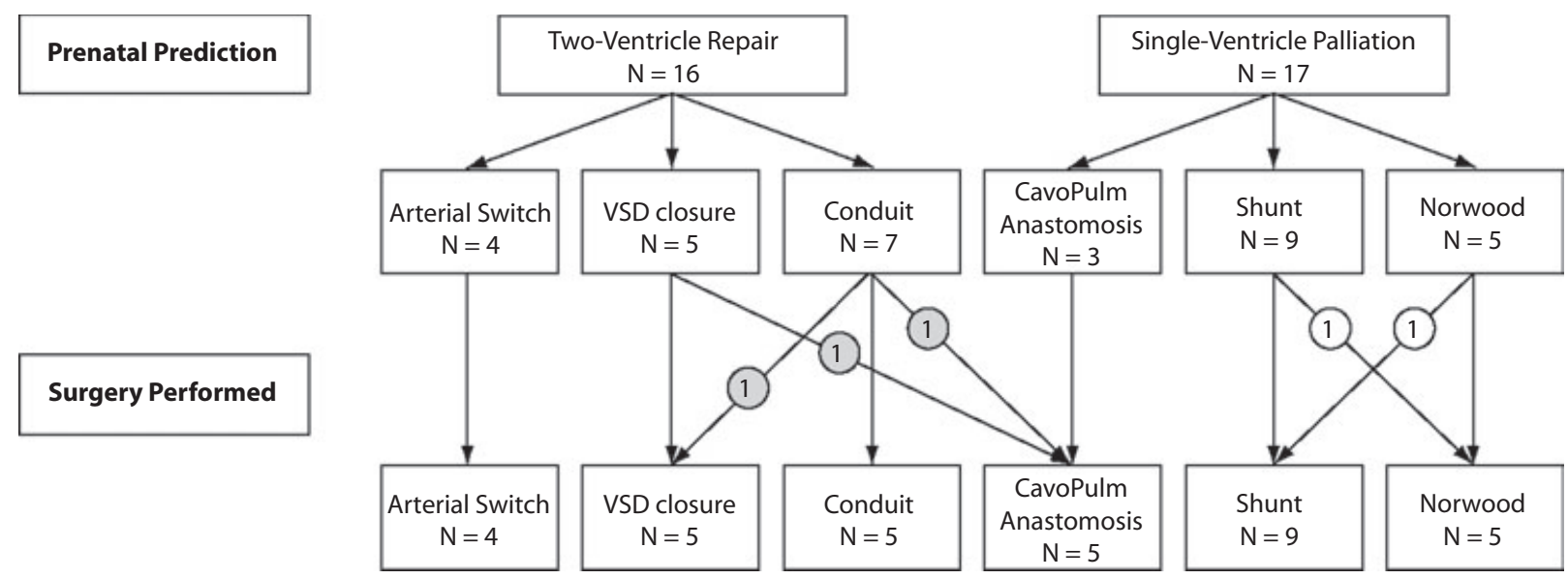

Figure 2. Prenatal surgical prediction compared with surgery performed. Numbers in circles represent number of patients who changed categories between prenatal prediction and surgery performed. CavoPulm indicates cavopulmonary; VSD, ventricular septal defect.

with placement of a right ventricle to pulmonary artery conduit; (3) arterial switch procedure with VSD closure; and (4) single-ventricle palliation including the Norwood procedure, aortopulmonary shunt placement, or cavopulmonary anastomosis as the initial surgery.

Accurate prenatal prediction of the type of surgery was made in $91 \%$ of the patients who had surgery. All those predicted prenatally to need a single-ventricle palliation ultimately required this type of surgery. Within this group, the initial stage of the palliation (aortopulmonary shunt, Norwood procedure, or cavopulmonary anastomosis) was correctly predicted in $88 \%$. Accurate prediction of the type of repair needed was made in 14 of the 16 patients $(81 \%)$ predicted to be candidates for 2 ventricular repair, with 2 undergoing singleventricle palliation. Of the 8 patients given postnatal diagnoses other than DORV, accurate counseling regarding the type of surgery to be performed was given in all 6 who had surgery; the other 2 patients had major extracardiac anomalies and did not undergo surgery.

Overall patient outcomes, regardless of postnatal diagnosis, are shown in Figure 3. Excluding terminations, neonatal survival ( $>28$ days) for the remaining 43 patients was $72 \%$ with 2 in utero demises and 10 neonatal deaths. No clear cause of death was identified in the in utero demises, both of which occurred in the third trimester. Of live births, survival to hospital discharge was $76 \%$ with 8 preoperative deaths and 2 early postoperative deaths. Infants with extracardiac or chromosomal anomalies had a hospital survival rate of $50 \%$ compared with $92 \%$ survival in infants without additional anomalies $(P=.004)$. No significant difference in survival rates was found in patients with heterotaxy, atrioventricular canal defects, or pulmonary vein anomalies compared with patients without those additional lesions.

\section{Discussion}

We found improved diagnostic accuracy in fetal DORV as compared with prior studies that reported accuracy of $53-65 \% ., 5$ This may reflect improvements in echocardiographic technology over time. Discrepancies between pre- and postnatal diagnosis may be due to differences in the definition of DORV. ${ }^{6,7}$ One definition depends on the extent of posterior great vessel override over the right ventricle. The other is based on the absence of mitral-semilunar valve continuity or, put another way, the presence of bilateral subarterial conus tissue. Both definitions are based on anatomic findings that can be subtle on fetal echocardiogram.

Most patients in our series who were misdiagnosed with DORV by fetal echocardiogram were ultimately diagnosed with lesions in the same spectrum of disease. For example, 4 patients with prenatal diagnoses of DORV and subaortic VSD had postnatal diagnoses of tetralogy of Fallot (2 patients) and isolated VSD (2 patients). It could be argued that in these cases, the lesions are nearly the same physiologically as well as surgically. Nonetheless, it is important to recognize that patients with true DORV commonly have 
Gelebrteret al.

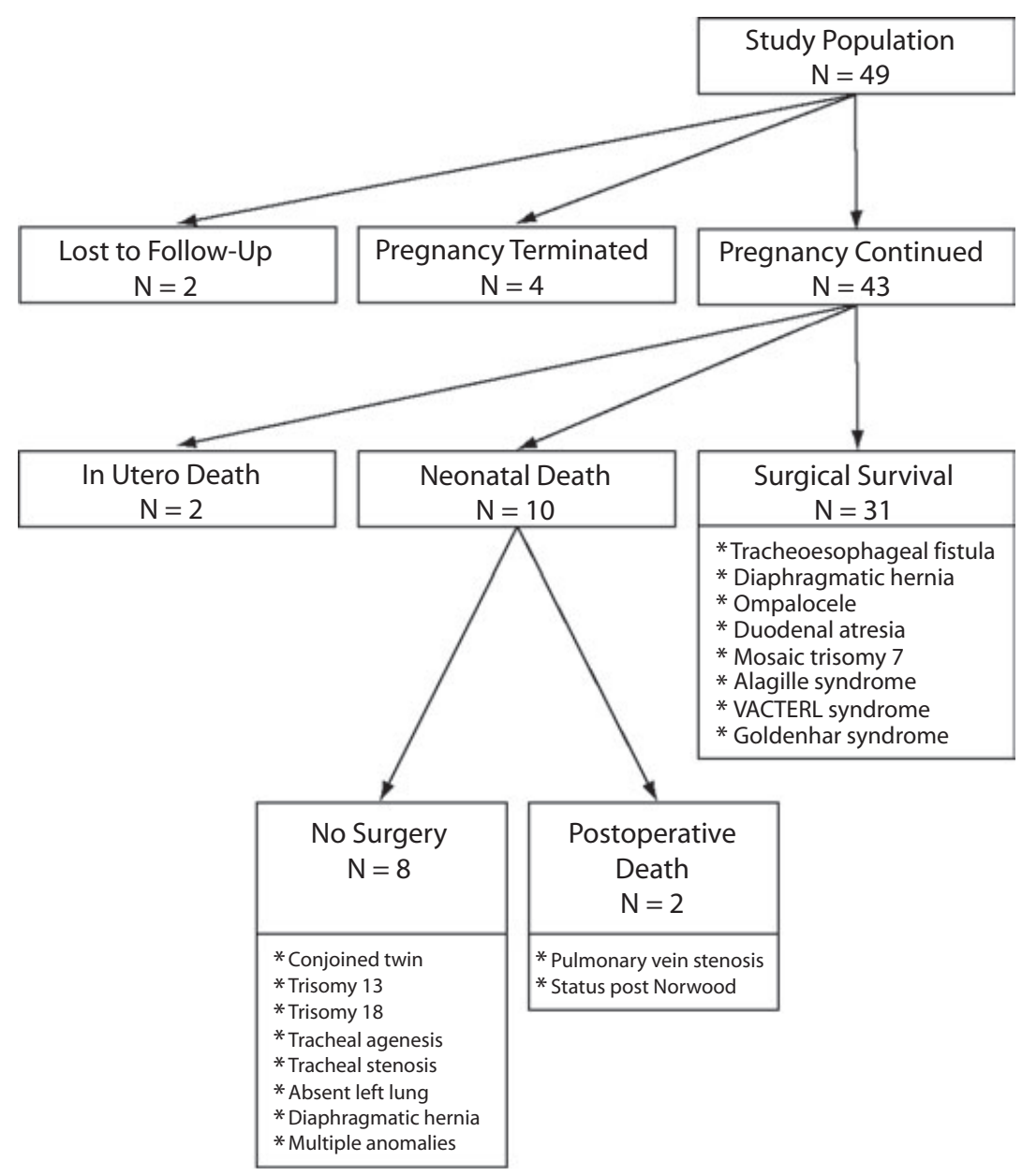

Figure 3. Outcomes for all patients, regardless of postnatal diagnosis, with associated major extracardiac anomalies listed.

complicating anatomical issues such as straddling atrioventricular valves, complex VSD anatomy, and extracardiac anomalies. As these issues may impact the prognosis, they should be specifically addressed when counseling the families of fetuses with suspected DORV.

Despite some inaccuracy in exact anatomic diagnosis, accurate counseling regarding the type of surgical repair needed was given in nearly all cases. Inaccurate predictions were made in 3 patients. In 1 case, the patient was predicted to need a repair involving a right ventricle to pulmonary artery conduit. Postnatally, the patient was able to have a "tetralogy-type" repair with a transannular patch. The second patient had a mildly hypoplastic mitral valve seen on fetal echocardiogram at 32 weeks of gestation; postnatal evaluation showed more significant hypoplasia of the mitral valve and left ventricle, requiring single-ventricle palliation. The third patient was diagnosed prena- tally with 2 normal-sized ventricles and a VSD remote from the great arteries. Despite prenatal prediction that a 2 -ventricle repair might be possible, the location of the VSD and the presence of multiple straddling tricuspid valve attachments ultimately precluded 2-ventricle repair.

As DORV is associated with additional lesions, such as atrioventricular valve abnormalities, a high level of diagnostic detail is necessary of fetal echocardiography to make accurate predictions regarding prognosis. All the patients with alternative, but related, postnatal diagnoses had accurate prenatal predictions regarding type of surgery needed, suggesting that enough anatomic details were correctly determined to allow appropriate counseling.

We report improved survival for infants with prenatally diagnosed DORV as compared with previous studies. In our series, neonatal survival of fetuses with confirmed DORV was $84 \%$ when 
terminations were excluded; for the entire study population the neonatal survival was $72 \%$. This is an improvement on the 50-60\% survival reported in other studies of fetal diagnosis of DORV. ${ }^{3-5}$ Four of the 5 deaths of neonates with a confirmed diagnosis of DORV were in patients with singleventricle lesions and major extracardiac malformations. Support was withdrawn in 3 patients because of major extracardiac anomalies, the fourth died of complications of a diaphragmatic hernia repair, and the fifth died of congenital pulmonary vein stenosis following "tetralogy type" repair.

The retrospective nature of our study does create limitations. Our study population included a high proportion of patients with single-ventricle lesions, which reflects referral patterns at our institution. The size of our study population was relatively small, which did not allow for detailed subgroup analysis; the numbers within each subgroup were too small to identify any predictors of outcome. However, to our knowledge, this is the largest series of fetal diagnosis of DORV reported to date.

\section{Conclusion}

Fetal diagnosis of DORV continues to be difficult given the variability in anatomy. Careful fetal echocardiography can determine essential details such as ventricular adequacy, great artery orientation, great artery size, and VSD location. By determining these important anatomic details, accurate counseling regarding the type of surgery needed was given in most cases. Survival for the neonate with DORV is better than has been previously reported, but is still poor in infants with major extracardiac malformations.

Corresponding Author: Sarah Gelehrter, MD, L1242 Women's Hospital Box 0204, 1500 E. Medical Center Drive, Ann Arbor, MI 48109-0204, USA. Tel: (+1) 734-936-6703; Fax: (+1) 734-936-9470; E-mail: sgelehrt@umich.edu

Accepted for publication September 27, 2006.

\section{References}

1 Allan LD, Sharland GK, Milburn A, et al. Prospective diagnosis of 1006 consecutive cases of congenital heart disease in the fetus. $7 \mathrm{Am}$ Coll Cardiol. 1994;23:1452-1458.

2 Forbus GA, Atz AM, Shirali GS. Implications and limitations of an abnormal fetal echocardiogram. $\mathrm{Am}$ 7 Cardiol. 2004;94:688-689.

3 Paladini D, Rustico M, Todros T, et al. Conotruncal anomalies in prenatal life. Ultrasound Obstet Gynecol. 1996;8:241-246.

4 Smith RS, Comstock CH, Kirk JS, Lee W, Riggs T, Weinhouse E. Double-outlet right ventricle: an antenatal diagnostic dilemma. Ultrasound Obstet Gynecol. 1999;14:315-319.

5 Tometzki AJ, Suda K, Kohl T, Kovalchin JP, Silverman NH. Accuracy of prenatal echocardiographic diagnosis and prognosis of fetuses with conotruncal anomalies. 7 Am Coll Cardiol. 1999;33: 1696-1701.

6 Walters HL, Mavroudis C, Tchervenkov CI, Jacobs JP, Lacour-Gayet F, Jacobs ML. Congenital Heart Surgery Nomenclature and Database Project: double outlet right ventricle. Ann Thorac Surg. 2000;69: S249-S263.

7 Anderson RH, McCarthy K, Cook AC. Double outlet right ventricle. Cardiol Young. 2001;11:329-344. 